\title{
SISTEMA DE COGENERACIÓN CON TURBINA DE GAS
}

\section{APLICACIÓN AL CALENTAMIENTO INDIRECTO DE AIRE}

\section{$\underline{\text { PARA EL SECADO DE MALTA }}$}

\author{
Autor: Prof. Ing. Alberto Fushimi \\ Tesis presentada para el grado de \\ MAGISTER EN INGENIERÍA \\ Departamento Mecánica, Facultad de Ingeniería, Universidad Nacional de la Plata \\ Abril de 1999 \\ Director de Tesis: \\ Prof. Dr. (UNESP) José Luz Silveira \\ Departamento de Energía, Facultad de Ingeniería de Guaratinguetá (FEG) \\ Universidad Estatal Paulista (UNESP), Sao Paulo, Brasil. \\ $\underline{\text { Co Director de Tesis }}$ \\ Prof. Ing. Pablo José Ringegni \\ Departamento Aeronáutica, Facultad de Ingeniería, Universidad Nacional de La Plata
}

$\underline{\text { Jurados de Tesis: }}$

Prof. Dr. (UBA) Carlos Marschoff

Prof. Dr. (Princeton University) Gautam Dutt. 